\title{
Myocardial migration by fibroblast progenitor cells is blood pressure dependent in a model of angll myocardial fibrosis
}

\begin{abstract}
Nicole L Rosin ${ }^{1}$, Mryanda Sopel ${ }^{1}$, Alec Falkenham ${ }^{1}$, Tanya L Myers ${ }^{2}$ and Jean-Francois Légaré1,2,3
Activation of the renin-angiotensin system (RAS) is thought to promote myocardial fibrosis. However, it is unclear whether this physiological fibrotic response results from chronic hemodynamic stress or from direct cellular signaling. Male C57B/6 mice were randomly assigned to receive angiotensin II (AngII) $\left(2.0 \mu \mathrm{gg}^{-1} \mathrm{~min}^{-1}\right)$, Angll+hydralazine $\left(6.9 \mu \mathrm{gg}^{-1} \mathrm{~min}^{-1}\right)$ or saline (control) via osmotic pumps for 7 days. Blood pressure was measured via noninvasive plethysmography. Hearts were harvested and processed for analysis. Cellular infiltration and collagen deposition were analyzed using histological staining. Molecular mediators were assessed using quantitative RT-PCR. As previously described, animals that received Angll developed hypertension and multifocal cellular infiltration by $\mathrm{SMA}^{+} / \mathrm{CD} 133^{+}$fibroblast progenitors followed by collagen deposition. The coadministration of hydralazine with Angll completely inhibited the hypertensive effects of Angll $(P \leqslant 0.01)$ and resulted in minimal cellular infiltration and minimal collagen deposition. These findings were in the context of persistent RAS activation, which was evidenced by elevation in serum aldosterone levels in animals that received Angll or Angll+hydralazine compared with animals that received saline. At the molecular level, infusion of Angll resulted in the significant upregulation of profibrotic factors (connective tissue growth factor-7.8 \pm 0.7 fold), proinflammatory mediators (TNF $\alpha-4.6 \pm 0.8$ fold; IL-1 $\beta-6.4 \pm 2.6$ fold) and chemokines (CCL2-3.8 \pm 1.0 fold; CXCL12-3.2 \pm 0.4 fold), which were inhibited when hydralazine was also infused. We provide evidence that myocardial infiltration by fibroblast progenitor cells secondary to Angll and the resultant fibrosis can be prevented by the addition of hydralazine. Furthermore, the beneficial effects of hydralazine were observed while maintaining RAS activation, suggesting that the mechanism of fibrosis is blood pressure dependent.
\end{abstract}

Hypertension Research (2012) 35, 449-456; doi:10.1038/hr.2011.217; published online 19 January 2012

Keywords: fibrocytes; fibrogenesis; myocardium; progenitor cells

\section{INTRODUCTION}

There is growing evidence that the renin-angiotensin system (RAS), specifically angiotensin II (angII), is implicated in the development of myocardial fibrosis, a common pathological feature of many cardiovascular conditions. ${ }^{1-4}$ RAS activation and increased levels of AngII can be seen in patients with cardiovascular diseases, including atherosclerosis, hypertension, cardiac hypertrophy and heart failure. ${ }^{1,5,6}$ Conversely, inhibition of RAS activation by angiotensin receptor blockade has been shown to significantly improve patient survival and limit the progression of cardiac remodeling and heart failure. ${ }^{7}$

Investigations in small animals have confirmed that AngII exposure can result in myocardial fibrosis. ${ }^{8-15}$ Such experiments have supported a direct relationship between RAS activation via AngII and fibrosis. Animal models of fibrosis using AngII have proven very helpful in understanding some of the molecular events leading to the development of fibrosis. However, AngII is also a potent vasoconstrictor capable of causing significant hypertension, making it difficult to dissociate the effects of hypertension vs. AngII on the myocardium. In fact, whether hypertension or RAS activation is the mechanism responsible for remodeling has been a longstanding debate. ${ }^{9,16}$

Using a mouse model of AngII infusion, our laboratory has been able to characterize the early molecular and cellular events occurring in the myocardium within 1 week of exposure to AngII. We have shown that animals exposed to AngII develop hypertension and early infiltration ( 1 day) by fibroblast progenitor cells known as fibrocytes. ${ }^{17}$ We and others have also provided evidence that strategies to limit the migration of fibrocytes inhibit the development of fibrosis, supporting an effector role for these cells.

In this manuscript, we have utilized our experience with the AngIIinfusion model to better characterize early events in the development of fibrosis. Specifically, we evaluated the effects of RAS activation in the absence of hypertension using the antihypertensive drug hydralazine, which acts on smooth muscle cells as a vasodilator independent of the RAS system.

${ }^{1}$ Department of Pathology, Dalhousie University, Halifax, Nova Scotia, Canada; ${ }^{2}$ Department of Surgery, New Halifax Infirmary, Halifax, Nova Scotia, Canada and ${ }^{3}$ Department of Microbiology and Immunology, Dalhousie University, Halifax, Nova Scotia, Canada 


\section{METHODS}

\section{Animals}

All work was approved by Dalhousie University's, University Committee on Laboratory Animals. Male C57BL/6 mice, ranging from 7-8 weeks of age, were purchased from Jackson Laboratory (Bar Harbour, ME, USA) and were housed within the Medical Sciences Animal Care Facility at Dalhousie University. The mice were provided with food and water ad libitum for 1 week before experimentation. Animals were anesthetized with isoflurane (Baxter Healthcare, New Providence, NJ, USA) in oxygen, delivered by a Fortec vaporizer (Cyprane, Keighley, UK). When surgical levels of anesthesia were reached, a $1-2-\mathrm{cm}$ midscapular skin incision was made and a mini osmotic pump (Alzet, Palo Alto, CA, USA) was inserted subcutaneously. The incision was closed using 7-mm wound clips. Animals were randomly assigned to receive AngII $(n=10$, $2.0 \mu \mathrm{g} \mathrm{kg}^{-1} \mathrm{~min}^{-1}$, Sigma-Aldrich, Oakville, ON, Canada), AngII and hydralazine $\left(n=4,6.9 \mu \mathrm{g} \mathrm{kg}^{-1} \mathrm{~min}^{-1}\right.$, Sigma-Aldrich) or a vehicle control of saline $(n=10)$. The pumps remained in place for 7 days, during which the animals were provided with food and water ad libitum and observed for signs of morbidity. Before euthanization, blood pressure measurements were taken via the Coda2 noninvasive cuff system (Kent Scientific, Torrington, CT, USA) for a minimum of five consecutive measurements per animal. Experience in our laboratory and findings from others have shown that blood pressure measurements taken at the completion of the experiment reflect changes that have been stable over several days, supporting our approach to measure blood pressure at 7 days. ${ }^{18,19}$ Animals were then anesthetized, blood was collected and the hearts were harvested and divided along the short axis into three portions, including the base, middle and apical sections. The base portion was processed for histological examination, whereas the other two portions were snap frozen immediately for molecular analysis.

\section{ELISA for aldosterone}

The collected blood was allowed to clot at room temperature, and the serum was collected after the sample was centrifuged at $600 \times \mathrm{g}$ for $10 \mathrm{~min}$. The samples were aliquoted and stored at $-80{ }^{\circ} \mathrm{C}$ until required for the ELISA assay. Serum aldosterone levels were assessed using a standard ELISA kit (Cayman Chemical Company, Ann Arbor, MI, USA) as per the manufacturer's instructions. Briefly, serum samples were diluted 1:4 and plated out in triplicate, along with recommended controls. The plate was covered and incubated for $18 \mathrm{~h}$ at $4{ }^{\circ} \mathrm{C}$. The plate was then developed for $1.5 \mathrm{~h}$ while shaking and was read at $420 \mathrm{~nm}$. The total concentrations for each sample were calculated, averaged and compared between groups.

\section{Histological analysis}

The hearts were fixed in $10 \%$ formalin, embedded in paraffin and serially sectioned $(5 \mu \mathrm{m})$. The basic myocardial histology and cellular infiltration were examined using heart cross-sections stained with hematoxylin and eosin. A blinded observer quantified the infiltrating cells by counting the number of grids affected within an image of an entire heart cross-section at $\times 5$ magnification (one section per animal), based on a previously published gridscoring method to quantify the degree of cellular infiltration between groups. ${ }^{17}$

\section{Collagen deposition}

Collagen detection was accomplished using Sirius red and fast green stains and quantified as previously described. ${ }^{17}$ Briefly, slides were examined under a microscope with the $\times 10$ objective and areas of collagen deposition were captured with a digital camera. Image analysis software was used to quantify the amount of tissue that was positive for Sirius red. Four images per section from each individual animal were averaged, and the experimental groups were compared with control animals.

\section{Immunohistochemistry}

Immunohistochemistry for CD133 (Abcam, Cambridge, MA, USA), $\alpha$-smooth muscle actin (SMA; Sigma-Aldrich), and Ki67 (Sigma-Aldrich) was performed as previously described. ${ }^{17}$ Briefly, endogenous peroxidases and nonspecific staining were blocked to prevent nonspecific staining. Each section was incubated with the relevant antibody followed by the relevant biotin-conjugated secondary antibody (Vector, Burlington, ON, Canada). The antibody complexes were then conjugated to an avidin-biotin complex (Vectastain $\mathrm{ABC}$
Table 1 Primer sequences of target genes

\begin{tabular}{lll}
\hline Gene symbol & Forward primer & Reverse primer \\
\hline CCL2 & CCAGCCAACTCTCACTGAAGC & AGCTCTCCAGCCTACTCATTGG \\
Colla1 & GTGGAGGGATTACACGAAGC & GTGGAGGGATTACACGAAGC \\
CTGF & TCAACCTCAGACACTGGTTCG & TAGAGCAGGTCTGTCTGCAAGC \\
CXCL12 & GTAGAATGGAGCCAGACCATCC & ATTCGATCAGAGCCCATAGAGC \\
IL $1 \beta$ & TCCTCGGCCAAGACAGGTCGCT & CCCCCACACGTTGACAGCTAGGT \\
TNF $\alpha$ & TCTCATGCACCACCATCAAGGACT & ACCACTCTCCCTTTGCAGAACTCA \\
HIF-1 & AACAGTCCCTCTGTAGTTGTGG & TAGCGACAAAGTGCATAAAACC \\
$18 S$ & TCAACTTTCGATGGTAGTCGCCGT & TCCTTGGATGGGTAGCCGTTTCT \\
\hline
\end{tabular}

Forward and reverse primers $\left(5^{\prime}-3^{\prime}\right.$ orientation) used for qRT-PCR.

kit; Vector) and developed using 3,3' diaminobenzidine as the chromogen (DakoCytomation, Burlington, ON, Canada). Light microscopy was performed and pictures were captured and analyzed in Adobe Photoshop 5.0 (San Jose, CA, USA).

\section{Relative quantitative polymerase chain reaction (qRT-PCR)}

Using the TRIzol reagent (Gibco-BRL, Gaithersburg, MD, USA), RNA was isolated from the snap frozen myocardium as per the manufacturer's protocol. First strand cDNA was synthesized from $1 \mu \mathrm{g}$ of total RNA using an iScript cDNA Synthesis Kit (Bio-Rad, Hercules, CA, USA).

Relative qRT-PCR was completed using $12.5 \mathrm{ng}$ of input cDNA with $0.5 \mu \mathrm{M}$ of each of the forward and reverse primers, and $1 \times$ iQ SYBR Green Supermix (Bio-Rad) was subjected to qRT-PCR using an iQ5 Multicolor Real-Time PCR Detection System thermocycler (Bio-Rad). Standard curves for efficiency and no-template control samples were run along with the samples during thermocycling. A melting curve was performed after thermocycling was complete to ensure target specificity. The primers were designed against the mRNA sequence of the selected growth factor or cytokine, as shown in Table 1. Expression was normalized to the 18 s ribosomal gene using the Pfaffl method.

\section{In vitro-primary fibroblast isolation and proliferation assay}

Mouse skin fibroblasts were isolated from ear clippings. The clippings were mechanically diced and enzymatically digested with collagenase type II (1.63 $\mathrm{mg} \mathrm{ml}^{-1}$ in Dulbecco's modified essential media) for $30 \mathrm{~min}$ at $37^{\circ} \mathrm{C}$ with $5 \% \mathrm{CO}_{2}$ (Invitrogen, Burlington, ON, Canada). The isolated cells were plated on $0.1 \%$ gelatin (Sigma) coated tissue culture flasks and allowed to grow to $80 \%$ confluence before use. All assays were performed using isolated cells before five passages.

A BrdU assay for the assessment of relative proliferation was completed according to the manufacturer's protocol (Millipore, Billerica, MA, USA). In brief, primary fibroblasts were plated on 96-well culture plates, allowed to adhere overnight and serum starved for $24 \mathrm{~h}$ before treatment. The cells were then treated with either saline as a control, AngII (100 nM), hydralazine ( $40 \mathrm{nM})$ or AngII+hydralazine for $6 \mathrm{~h}$, at which point the BrdU reagent was added to the culture plate. After $24 \mathrm{~h}$ from the time of treatment, the plates were fixed, dried and stored at $4{ }^{\circ} \mathrm{C}$ until analysis. Plates were then incubated with primary antiBrdU antibody, washed, incubated with secondary biotin-conjugated anti-goat antibody, washed, incubated with substrate, stopped and read at $490 \mathrm{~nm}$.

\section{Statistical analysis}

One-way ANOVAs were completed on all quantitative data, using the Newman-Keuls post-test to compare the experimental groups to the saline control. Our level of significance was set as $P \leqslant 0.05$. All statistical calculations were computed using GraphPad Prism 4 software (La Jolla, CA, USA).

\section{RESULTS}

\section{Model establishment}

The animals were randomly assigned to one of three experimental groups and infused with one of the following: (a) AngII 

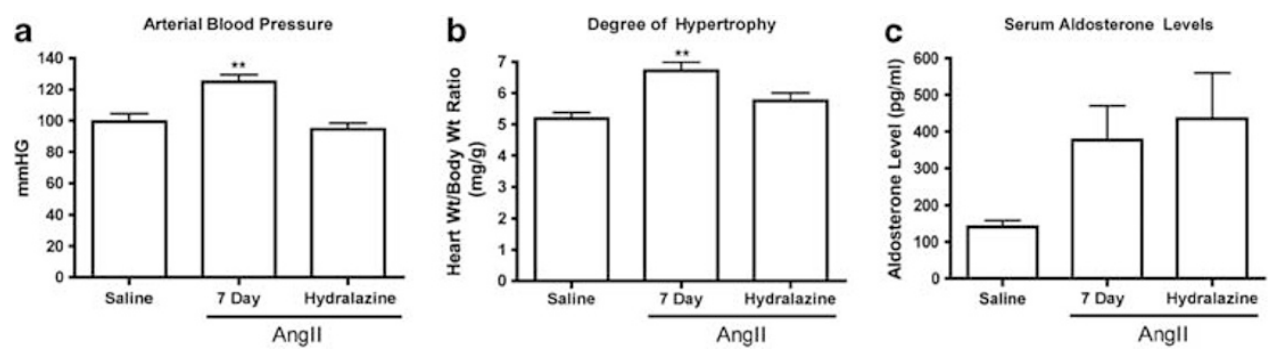

Figure 1 Establishing the model. Hemodynamic measurements were taken via a tail cuff blood pressure system (7 days) and compared between groups, with systolic blood pressure findings shown as mean \pm s.d. (a). The cardiac mass index was expressed as heart weight/body weight ratio at 7 days (b). Serum levels of aldosterone at 7 days were measured by ELISA and expressed as $\mathrm{pg} \mathrm{ml}^{-1}$ (c). ${ }^{* *} P<0.01$.

$\left(2.0 \mu \mathrm{g} \mathrm{kg}^{-1} \mathrm{~min}^{-1}, n=10\right)$; (b) AngII with hydralazine $\left(6.9 \mu \mathrm{g} \mathrm{kg}^{-1}\right.$ $\left.\min ^{-1} ; n=4\right)$ or (c) saline (control, $n=10$ ). Infusion of AngII, a known vasoconstrictor, resulted in a significant increase in mean arterial blood pressure compared with the saline control (Figure 1). Coadministration of AngII with hydralazine allowed normalization of the blood pressure to levels similar to those of the saline control animals (Figure 1a). Furthermore, hydralazine also inhibited the myocardial hypertrophic effects of AngII, as the AngII+hydralazine animals had a significantly lower heart weight/body weight ratio (Figure 1b), which was comparable to that of the saline controls (AngII, $6.7 \pm 0.3 \mathrm{mg} \mathrm{g}^{-1}$; AngII+hydralazine, $5.7 \pm$ $0.2 \mathrm{mg} \mathrm{g}^{-1}$; saline, $5.2 \pm 0.2 \mathrm{mg} \mathrm{g}^{-1}$ ). Serum levels of aldosterone were evaluated using a standard ELISA and showed that AngII and AngII+hydralazine animals had elevated serum aldosterone levels compared with the saline controls, although significance was not reached (Figure 1c). Taken together, our findings suggest that the hypertensive effects of AngII can be controlled with the antihypertensive agent hydralazine while maintaining RAS activation, thus creating a model with which to examine the early cellular and molecular events occurring in these animals.

\section{Myocardial fibrosis}

The degree of myocardial fibrosis in experimental animals was determined by quantifying the collagen depositions within the myocardium using heart sections stained with Sirius red, as previously described. ${ }^{17}$ AngII infusion resulted in multifocal areas of extensive collagen deposition compared with saline-infused control animals $(P<0.01$; Figures $2 \mathrm{a}$ and $\mathrm{b})$. When hydralazine was also infused, there was a significant reduction in collagen deposition evident within the myocardium, which approached levels seen in the saline controls, expressed as the percentage surface of an area of myocardium affected $(P<0.01$; Figures $2 \mathrm{c}$ and $\mathrm{d})$. These findings were confirmed by evidence of significant collagen-1 transcript upregulation in AngII animals $(103.0 \pm 36.2$ fold), with levels returning to baseline when hydralazine was coadministered $(P<0.01$; Figure $2 \mathrm{e})$.

\section{Cellular infiltration}

Previous work from our laboratory has shown that myocardial fibrosis is temporally preceded by the infiltration of fibroblast progenitor cells into the myocardium. ${ }^{17}$ In the present experiment, AngII exposure resulted in multi-focal areas of cellular infiltration and myocyte loss compared with saline controls (Figures $3 \mathrm{a}$ and $\mathrm{b}$ ). When the animals were given AngII in combination with hydralazine, minimal cellular infiltration was identified after 7 days of exposure (Figure 3c). We have adapted a grid-scoring method to quantify the degree of cellular infiltration between groups, confirming that significant myocardial cellular infiltration was evident in AngII-exposed animals and was inhibited by hydralazine $(P<0.01$; Figure $3 \mathrm{~d})$. The cells that were observed to infiltrate the myocardium were mononuclear in appearance, with no evident polymorphonuclear cells. Immunohistochemistry was used to confirm that a significant proportion of the cells observed in AngII-exposed animals were $\mathrm{SMA}^{+} / \mathrm{CD} 133^{+}$fibrocytes, as previously reported (Figure 4$).{ }^{17}$ In contrast, animals that received both AngII and hydralazine had minimal infiltration by $\mathrm{SMA}^{+}$/ $\mathrm{CD}_{133^{+}}$cells. Taken together, our findings confirm that myocardial fibrosis is dependent upon the accumulation of $\mathrm{SMA}^{+} / \mathrm{CD} 133^{+}$ fibrocytes before extracellular matrix deposition and that this process is blood pressure dependent, given that hydralazine can inhibit this response.

\section{Myocardial profibrotic cytokine environment}

Transcript levels of a well-known profibrotic factor, connective tissue growth factor (CTGF), were significantly increased in the myocardium at 7 days ( $7.8 \pm 0.8$ fold) after AngII exposure ( $P=0.008$; Figure 5). The coadministration of AngII with hydralazine resulted in a significant reduction in CTGF back to baseline levels seen in the saline control animals (Figure 5). Similar findings were observed for the proinflammatory cytokines TNF $\alpha$ (4.6 \pm 0.8 fold) and IL1 $\beta(6.4 \pm 2.6$ fold) in animals receiving AngII, with a return to baseline in the animals coadministered AngII+hydralazine $(P<0.05$; Figure 5$)$.

\section{Myocardial chemokine expression associated with fibrocyte migration}

To investigate which factors in the myocardial microenvironment may be recruiting the infiltrating cells, we used relative qRT-PCR to assess the transcript levels of the cytokines that are chemotactic for fibroblast progenitor cells, CXCL12 and CCL2. ${ }^{20,21}$ There was a significant increase in CXCL12 mRNA ( $3.2 \pm 0.4$ fold; $P=0.008$ ) expression in the myocardium of animals exposed to AngII at 7 days compared with the baseline expression of the normalized housekeeping gene (Figure 6). Similarly, there was a significant increase in CCL2 mRNA expression in the myocardium of AngII-exposed animals $(3.8 \pm 1.0$ fold; $P=0.015)$. The addition of hydralazine resulted in complete inhibition of the upregulation of CXCL12 and CCL2 in the myocardium, resulting in levels similar to those seen in the control animals (Figure 6).

\section{Effects of hydralazine at the cellular level}

The effects of hydralazine, other than those resulting from vasodilation and blood pressure reduction, were also investigated. We first looked at the induction of the transcription factor hypoxia-inducible factor 1 (Hifl), which has been suggested as a downstream effector of hydralazine. The transcript levels, measured by qRT-PCR, were significantly increased after 7 days of AngII exposure (5.0 \pm 0.8 fold; 

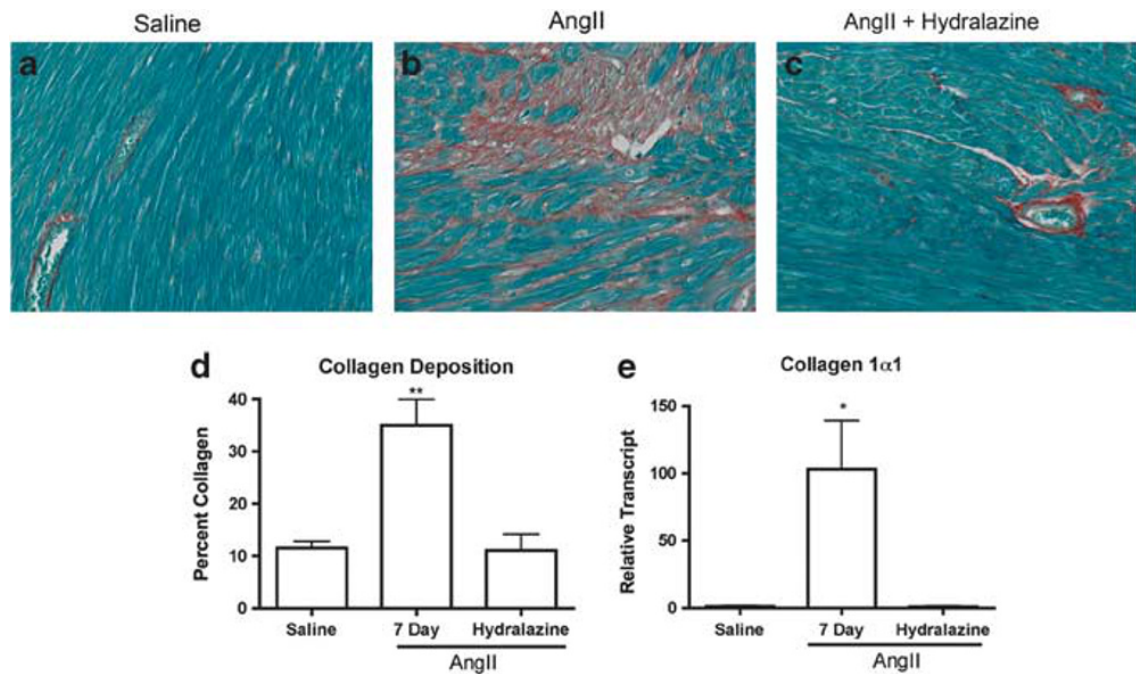

Figure 2 Myocardial fibrosis. The collagen deposition in heart sections from the experimental groups was assessed using a standardized Sirius red and fast green stain. Representative sections are shown from control animals (a), 7 days Angll-exposed animals (b) and 7 days Angll+hydralazine-exposed animals (c). Collagen in the heart sections was quantified using image analysis software and expressed as the percentage area affected (d). Collagen deposition was also quantified using whole heart extracts and processed for qRT-PCR for pro-collagen-1 (e). Images were captured at $\times 25$. ${ }^{* *} P<0.01$, ${ }^{*} P<0.05$

a
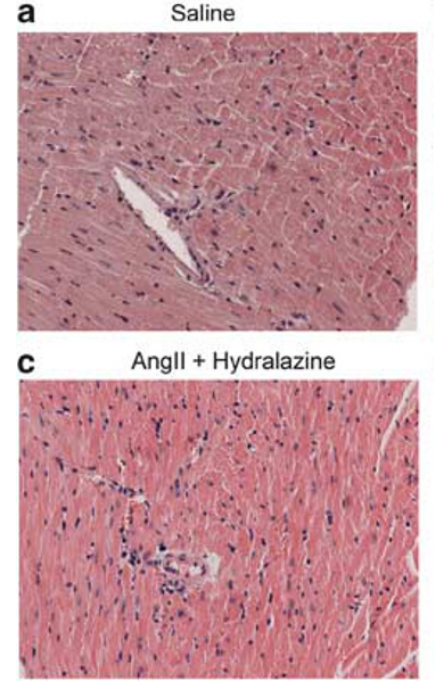

b

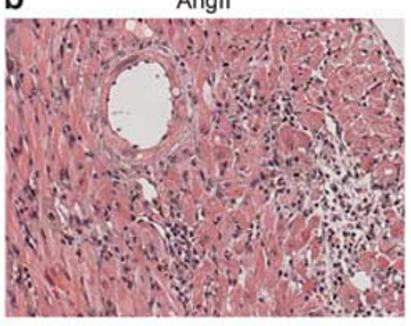

d

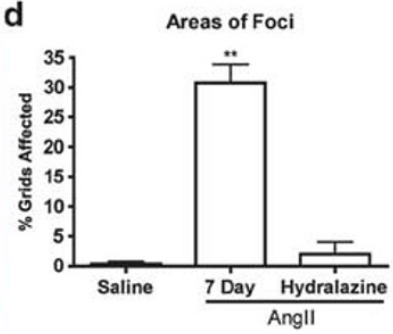

Figure 3 Cellular infiltration. The hearts from animals receiving the saline control (a), Angll for 7 days (b) or Angll+hydralazine for 7 days (c) were stained with hematoxylin and eosin. The quantification of infiltrating cells was performed using a modified grid-analysis technique, comparing the number of grids affected from a representative cross section of the myocardium (heart) taken at low power ( $\times 5$ magnification) from each group (d). Images were captured at $\times 25$. ${ }^{* *} P<0.01$.

$P=0.016$ ); however, when hydralazine was administered in combination with AngII, the levels of Hif1 were not elevated but were similar to baseline levels seen in the control animals (Figure 7).

Our previous work has shown that in areas of myocardial infiltration by fibrocytes, a significant number of these cells are proliferating. ${ }^{17}$ We therefore investigated the effects of hydralazine on proliferation both in vivo and in vitro. As previously described, the infiltrating $\mathrm{SMA}^{+} / \mathrm{CD} 133^{+}$cells were shown to have a high proliferative index, as demonstrated by strong positivity for Ki67 after AngII exposure. In contrast, animals that received both AngII and
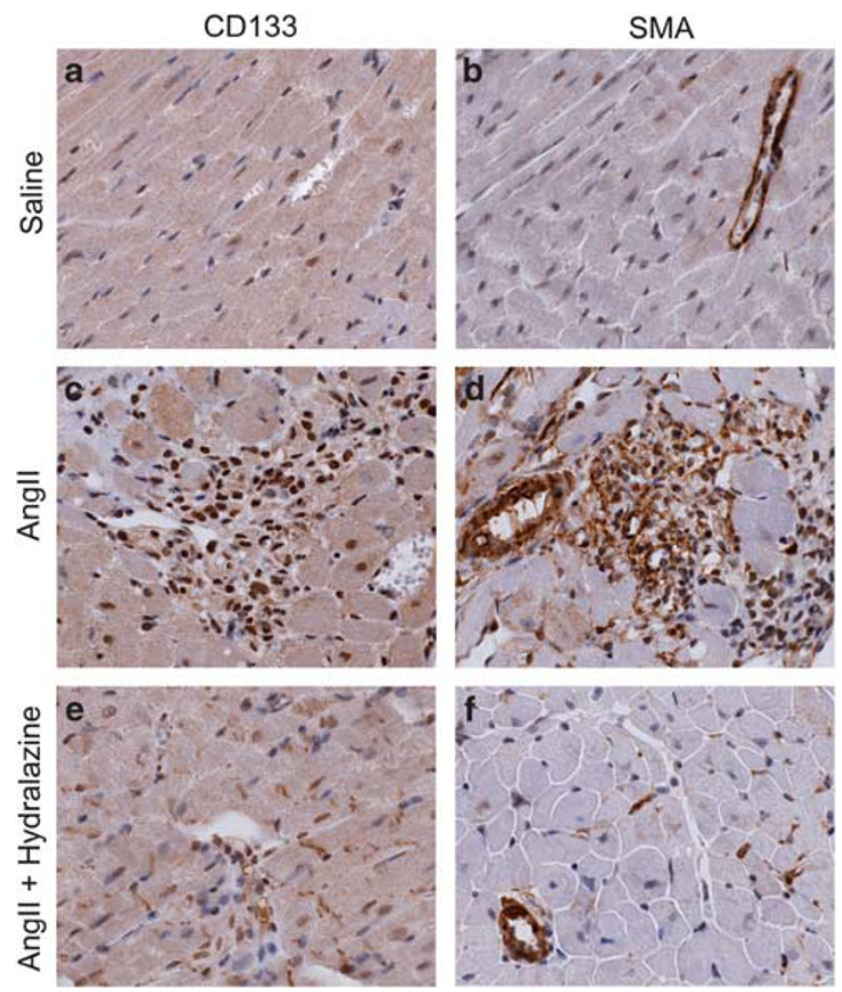

Figure 4 Immunohistochemical staining. Immunohistochemistry for CD133 (a, c, e) and SMA (b, d, f) was used to identify positive cellular infiltrate in heart sections. Representative sections from animals receiving the saline control (a, b), Angll for 7 days (c, d) or Angll+hydralazine for 7 days (e, f) are shown. Images were captured at $\times 63$.

hydralazine had a significant reduction in the positivity for Ki67, suggesting that hydralazine may affect the proliferation of fibrocytes (Figure 8). To confirm these findings we used an in vitro assay with primary fibroblasts, assessing the relative proliferation of primary 

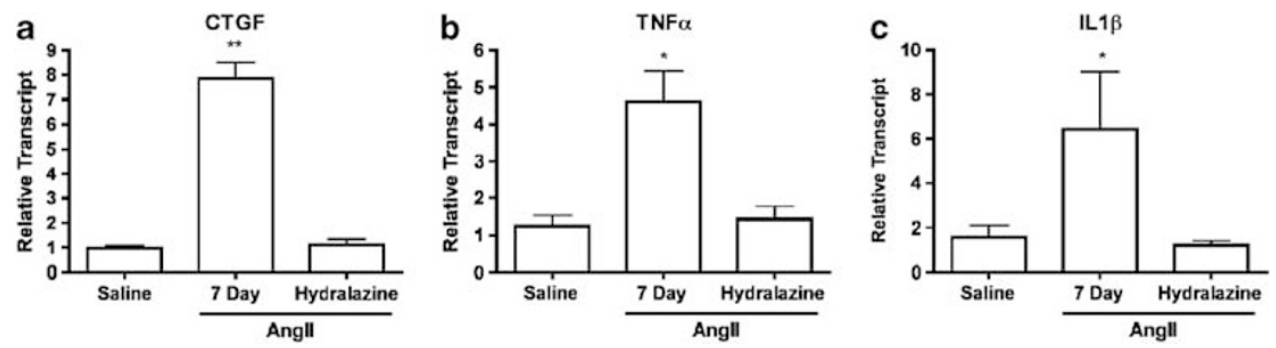

Figure 5 Profibrotic microenvironment. qRT-PCR was used to assess the myocardial expression of CTGF (a), TNF $\alpha$ (b) and IL-1 $\beta$ (c) transcripts relative to the housekeeping gene. ${ }^{*} P<0.01,{ }^{*} P<0.05$.
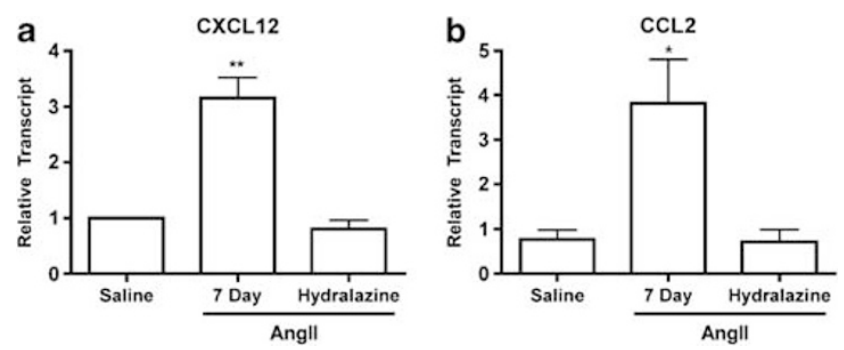

Figure 6 Chemokine expression. qRT-PCR was used to assess myocardial expression of CXCL12 (a) and CCL2 (b) transcript levels relative to the housekeeping gene $(\mathrm{E})$. ${ }^{* *} P<0.01,{ }^{*} P<0.05$.

fibroblast cultures as determined by BrdU incorporation. Our in vitro findings suggested that the proliferation of fibroblasts after hydralazine exposure, in the presence or absence of AngII, was significantly reduced by $30.6 \%$ from the level of the saline controls (Figure 8 ). Similar findings were obtained with an MTT assay (results not shown). Taken together, these results indicate that hydralazine may have some effect at the cellular level that could modulate the development of fibrosis, independently of blood pressure reduction.

\section{DISCUSSION}

AngII infusion models have been well described and are known to result in increased blood pressure, myocardial hypertrophy and a characteristic extracellular matrix deposition within the myocardium that is typical of myocardial fibrosis. ${ }^{20,22,23}$ In the mouse model used here, the physiological response to AngII resulted in blood pressure elevation and the early development of myocardial hypertrophy within 7 days of exposure. Using this model, we attempted to create a situation in which RAS upregulation via AngII infusion was maintained without the resultant hypertensive response. We achieved this by coadministration of hydralazine.

Hydralazine was chosen based on its antihypertensive properties of lowering blood pressure in a dose-dependent manner without acting on AngII receptors. ${ }^{24,25}$ RAS upregulation was demonstrated in the animals that received AngII+hydralazine, as evidenced by a significant elevation in serum aldosterone, which is a downstream mediator and marker of RAS activation. Our primary observation using this model was that the addition of hydralazine to AngII resulted in normalization of the blood pressure and inhibition of the fibrosis that is normally seen in animals receiving AngII. Taken together, these results indicate that AngII and its effects on promoting myocardial fibrosis appear, at least in part, to be blood pressure dependent.

Studies from our laboratory and other research groups have recently identified bone-marrow-derived fibroblast progenitor cells as the primary cells recruited to the myocardium before the development

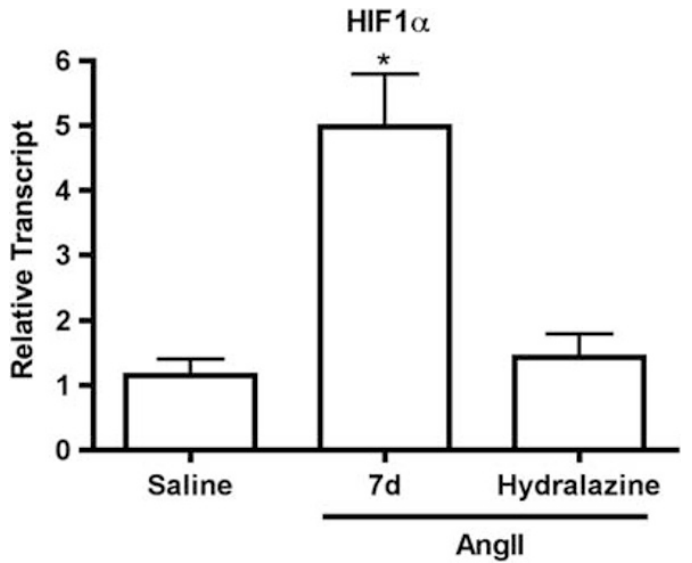

Figure 7 Transcription factor expression. qRT-PCR was used to assess the myocardial Hif1 transcript expression relative to the housekeeping gene. ${ }^{*} P<0.05$.

of fibrosis. ${ }^{17,26}$ We were able to show minimal infiltration by $\mathrm{SMA}^{+}$/ $\mathrm{CD}_{133^{+}}$fibrocytes in animals receiving AngII+hydralazine compared with animals receiving AngII. These findings support a direct relationship between cell migration and the development of fibrosis. Fully characterizing the mechanism by which AngII results in myocardial fibrosis is beyond the scope of this manuscript. However, using our model, in which RAS upregulation was maintained without hypertension, we explored key pathways previously described as potentially being involved in promoting fibrogenesis. We chose to assess CTGF as a profibrotic cytokine based on evidence that it is produced by cardiomyocytes, providing a mechanism by which AngII acts directly on the myocardium and promotes a profibrotic environment. AngII has been shown to increase CTGF expression within the myocardium and specifically in cardiomyocytes in vitro. ${ }^{27,28}$ CTGF also appears to be capable of inducing both profibrotic and proinflammatory gene expression in cultured cardiomyocytes. ${ }^{29}$ In fact, CTGF has been suggested to be the primary mediator of AngII-induced myocardial fibrosis. ${ }^{30}$ Our findings suggest that CTGF, which was significantly upregulated in AngII-exposed animals, was completely inhibited by the administration of hydralazine. Similar findings were observed with the classic proinflammatory cytokines TNF $\alpha$ and IL1 $\beta$. Although we provide no direct evidence that hydralazine inhibits the production of these cytokines, we provide strong evidence of an association between early fibrocyte migration and profibrotic cytokine production in the myocardium.

Interactions between chemokines and their respective receptors have emerged as key mechanisms in the directed migration of leukocytes and fibrocytes. ${ }^{31-35}$ Fibrocytes have previously been 

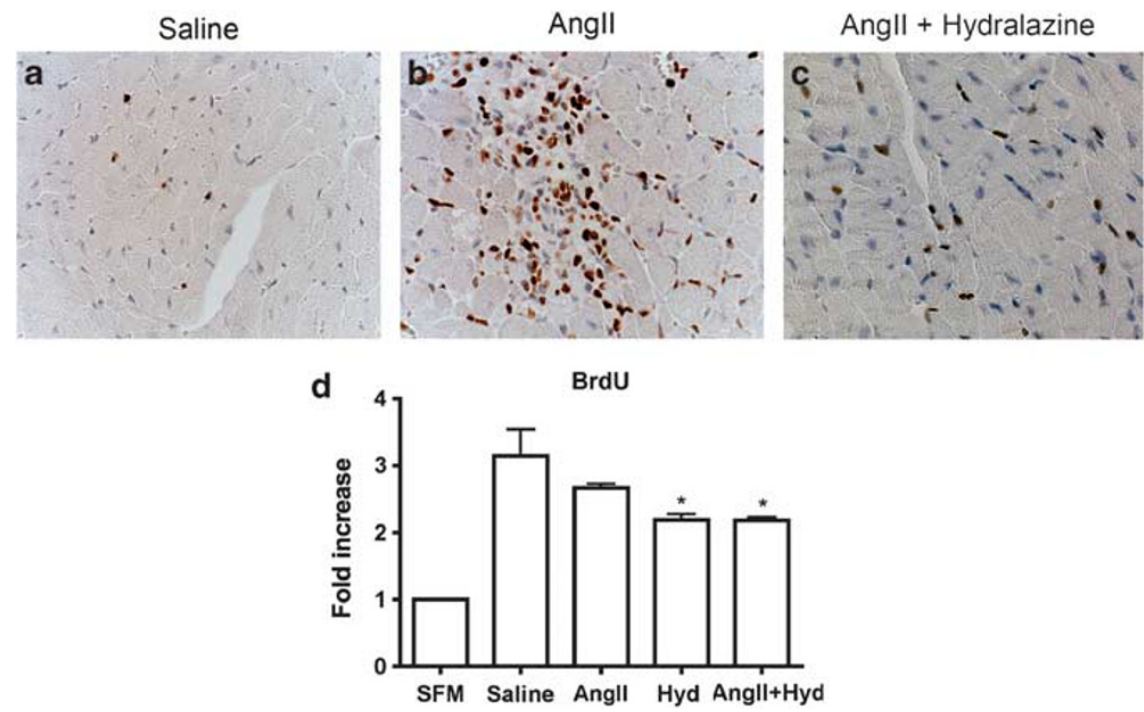

Figure 8 Cellular effects of hydralazine. Immunohistochemistry for Ki67 was used to illustrate the proliferative index of infiltrating fibrocytes. Representative sections from animals receiving saline (a), Angll (b) or Angll+hydralazine (c) are shown. Images were captured at $\times 63$. The BrdU assay for proliferation was used on primarily isolated fibroblasts after exposure to saline, Angll, hydralazine or Angll+hydyralazine (d). Relative proliferation was expressed as the fold increase compared with serum-free media. ${ }^{*} P<0.05$.

shown to express the chemokine receptors CCR7 and CXCR4. ${ }^{36-38}$ Recently, Haudek et al. ${ }^{26}$ was able to show that CCR2 has a critical role in regulating fibrocyte migration in a model of myocardial fibrosis. Using CCR2 $2^{-1-}$ animals, they were able to show a significant reduction in AngII-dependent fibroblast progenitor cell migration (fibrocytes) and a reduction in fibrosis. In the present manuscript, we did not attempt to modulate fibrocyte migration by blocking specific chemokines. However, our findings support previous studies by others that have suggested a key role for CXCL12 and CCL2 in regulating fibrocyte migration and accumulation.

It is generally assumed that fibroblasts and myofibroblasts are the cells responsible for extracellular matrix deposition. As such, there is a large body of literature that supports the direct effects of AngII, via the AT1 receptor, in promoting fibroblast proliferation, myofibroblast differentiation and fibrosis. ${ }^{12-15}$ We provide evidence that the interaction of AngII with its receptor may be sufficient, but not necessary, for fibrosis. Our findings are in keeping with other studies that have suggested that the effects of AngII on fibrosis are blood pressure dependent. $^{24,25,39}$ Some reports have even suggested that blood pressure can affect the migration of fibrocytes $\left(\mathrm{CD} 45^{+} / \mathrm{SMA}^{+}\right)$into the myocardium, but these reports did not elucidate a definitive mechanism. ${ }^{25}$ In contrast, transgenic mice that overexpress the ATla receptor and develop hypertrophy and fibrosis do so without developing hypertension, emphasizing the multifactorial pathways capable of promoting extracellular matrix deposition and fibrosis. ${ }^{40}$ However, these findings were based on transgenic mice that had abnormal RAS regulation based on overexpression of only the AT1 receptor. Data from spontaneously hypertensive models suggest that hydralazine was unable to fully inhibit the development of fibrosis, but these findings can be explained by mechanisms independent of RAS activation. ${ }^{41}$

In contrast to our findings in the present manuscript, there are examples in the literature of cases in which a reduction in blood pressure to normotensive levels did not abrogate organ damage, suggesting that fibrosis may be blood pressure independent under certain circumstances. ${ }^{41,42}$ However, some of these studies had other important differences that should be taken into consideration, such as the presence of metabolic syndrome using genetically mutated mice, in which obesity, dietary supplementation, glucose intolerance and hyperinsulinemia likely contributed to end organ damage. ${ }^{41}$ The same can be said for a model combining aldosterone administration, uninephrectomy and a high salt diet, in which increased load on the remaining kidney likely contributed to organ damage. ${ }^{42}$ Furthermore, hydralazine has traditionally been administered in the drinking water, whereas in the current study, we used a more direct administration of hydralazine through an osmotic mini-pump, providing a constant dosage over time. ${ }^{42,43}$ Our approach may have resulted in a higher effective dose, as the drug was delivered more directly to the circulation.

Some experimental studies have shown a beneficial effect of mineralocorticoid antagonists in reducing myocardial fibrosis that was suggested to be independent of blood pressure effects. These results have been explained by reductions in myocardial oxidative stress, suggesting that oxidative stress may be the common pathway by which AngII activation and hypertension can lead to myocardial fibrosis. ${ }^{44,45}$ Although we did not specifically examine oxidative stress, our findings suggest that aldosterone upregulation may not be sufficient for fibrosis, given the absence of fibrosis in AngII+hydralazine animals that had increased levels of aldosterone in their serum. Finally, the mechanisms should be considered by which hydralazine might reduce fibrosis, other than its antihypertensive effect. One mechanism that was recently suggested is that hydralazine may induce Hifl, which can in turn affect multiple genes as a transcription factor that mediates adaptive responses to injury, such as ischemia. ${ }^{46,47}$ Our findings support a significant upregulation of Hif1 with AngII exposure but, when coadministered with hydralazine, Hif1 levels returned to baseline. From our data, it is not possible to conclude that hydralazine has any direct effect on Hif1. We can conclude only that in animals without hypertension or myocardial histological changes that are receiving AngII+hydralazine, there appears to be no significant upregulation in mRNA encoding Hifl.

In the present manuscript, we attempted to separate RAS activation from hypertension and thereby identify potential mechanisms for the 
development of myocardial fibrosis. This separation was necessary because the downstream effects of RAS activation that lead to myocardial fibrosis are not fully characterized. Clinical trials published to date using RAS blocking agents, such as angiotensin receptor blockers and angiotensin converting enzyme inhibitors, have established a clear benefit for patients, with a significant reduction in the number of adverse cardiac events. ${ }^{7,9}$ This is in contrast to clinical studies that have used hydralazine and achieved similar reductions in blood pressure but failed to offer the same clinical benefit as RAS inhibition. ${ }^{48-50}$ In the present manuscript, we provide no explanation for this discrepancy. Taken together, these results suggest that blood pressure reduction may not be the sole factor influencing myocardial events such as fibrosis. The data obtained in the present study support the notion that although fibrosis appears to be partly dependent on blood pressure elevation, the antifibrotic properties of hydralazine may extend beyond its antihypertensive effects. We observed a reduction in fibrocyte and fibroblast proliferation in response to hydralazine both in vivo and in vitro. Although discerning the cellular mechanism responsible for this antiproliferative response is beyond the scope of this manuscript, our findings suggest that regulation of the antifibrotic properties of hydralazine, by mechanisms other than the vasodilator effects, should be considered.

In summary, we provide evidence that the effects of AngII on the development of myocardial fibrosis appear to be at least partly blood pressure dependent. We also provide evidence that blood pressure elevation seems necessary for early fibrocyte migration and for the development of a profibrotic environment, although the mechanisms for this are unclear at present.

\section{ACKNOWLEDGEMENTS}

The present work was supported in part by grants from the Nova Scotia Health Research Foundation (\#42311) and the Canadian Institute of Health Research (\#44122).

1 Kim S, Iwao H. Molecular and cellular mechanisms of angiotensin II-mediated cardiovascular and renal diseases. Pharmacol Rev 2000; 52: 11-34.

2 Towbin JA. Scarring in the heart-a reversible phenomenon? N Engl J Med 2007; 357: 1767-1768.

3 Ishiyama Y, Gallagher PE, Averill DB, Tallant EA, Brosnihan KB, Ferrario CM. Upregulation of angiotensin-converting enzyme 2 after myocardial infarction by blockade of angiotensin II receptors. Hypertension 2004; 43: 970-976.

4 de Resende MM, Kauser K, Mill JG. Regulation of cardiac and renal mineralocorticoid receptor expression by captopril following myocardial infarction in rats. Life Sci 2006; 78: 3066-3073.

5 Zhao Q, Ishibashi M, Hiasa K, Tan C, Takeshita A, Egashira K. Essential role of vascular endothelial growth factor in angiotensin II-induced vascular inflammation and remodeling. Hypertension 2004; 44: 264-270.

6 Brasier AR, Recinos 3rd A, Eledrisi MS. Vascular inflammation and the renin-angiotensin system. Arterioscler Thromb Vasc Biol 2002; 22: 1257-1266.

7 Schmieder RE, Hilgers KF, Schlaich MP, Schmidt BM. Renin-angiotensin system and cardiovascular risk. Lancet 2007; 369: 1208-1219.

8 Liu J, Yang F, Yang XP, Jankowski M, Pagano PJ. NAD(P)H oxidase mediates angiotensin II-induced vascular macrophage infiltration and medial hypertrophy. Arterioscler Thromb Vasc Biol 2003; 23: 776-782.

9 Billet S, Aguilar F, Baudry C, Clauser E. Role of angiotensin II AT1 receptor activation in cardiovascular diseases. Kidney Int 2008. 74: 1379-1384.

10 Harada K, Komuro I, Shiojima I, Hayashi D, Kudoh S, Mizuno T, Kijima K, Matsubara $\mathrm{H}$, Sugaya T, Murakami K, Yazaki Y. Pressure overload induces cardiac hypertrophy in angiotensin II type 1A receptor knockout mice. Circulation 1998; 97: 1952-1959.

11 Li HL, She ZG, Li TB, Wang AB, Yang Q, Wei YS, Wang YG, Liu DP. Overexpression of myofibrillogenesis regulator-1 aggravates cardiac hypertrophy induced by angiotensin II in mice. Hypertension 2007; 49: 1399-1408.

12 Dostal DE, Booz GW, Baker KM. Angiotensin II signalling pathways in cardiac fibroblasts: conventional versus novel mechanisms in mediating cardiac growth and function. Mol Cell Biochem 1996; 157: 15-21.

13 Sadoshima J, Izumo S. Molecular characterization of angiotensin II-induced hypertrophy of cardiac myocytes and hyperplasia of cardiac fibroblasts. Critical role of the AT1 receptor subtype. Circ Res 1993; 73: 413-423.
14 Crabos M, Roth M, Hahn AW, Erne P. Characterization of angiotensin II receptors in cultured adult rat cardiac fibroblasts. Coupling to signaling systems and gene expression. J Clin Invest 1994; 93: 2372-2378.

15 Zhou G, Kandala JC, Tyagi SC, Katwa LC, Weber KT. Effects of angiotensin II and aldosterone on collagen gene expression and protein turnover in cardiac fibroblasts. Mol Cell Biochem 1996; 154: 171-178.

16 Mehta PK, Griendling KK. Angiotensin II cell signaling: physiological and pathological effects in the cardiovascular system. Am J Physiol Cell Physiol 2007; 292: C82-C97.

17 Sopel MJ, Rosin NL, Lee TD, Legare JF. Myocardial fibrosis in response to Angiotensin II is preceded by the recruitment of mesenchymal progenitor cells. Lab Invest 2011; 91: 565-578.

18 Inaba S, Iwai M, Furuno M, Kanno H, Senba I, Okayama H, Mogi M, Higaki J, Horiuchi M. Temporary treatment with AT1 receptor blocker, valsartan, from early stage of hypertension prevented vascular remodeling. Am J Hypertens 2011; 24: 550-556.

$19 \mathrm{Li} \mathrm{C}$, Salisbury R, Ely D. Hydralazine reverses stress-induced elevations in blood pressure, angiotensin $\mathrm{II}$, testosterone, and coronary pathology in a social colony model. ISRN Pathol 2011 (doi:10.5402/2011/367280).

20 Haudek SB, Xia Y, Huebener P, Lee JM, Carlson S, Crawford JR, Pilling D, Gomer RH, Trial J, Frangogiannis NG, Entman ML. Bone marrow-derived fibroblast precursors mediate ischemic cardiomyopathy in mice. Proc Natl Acad Sci USA 2006; 103 : 18284-18289.

21 Chu PY, Mariani J, Finch S, McMullen JR, Sadoshima J, Marshall T, Kaye DM. Bone marrow-derived cells contribute to fibrosis in the chronically failing heart. Am J Pathol 2010; 176: 1735-1742.

22 Haudek SB, Trial J, Xia Y, Gupta D, Pilling D, Entman ML. Fc receptor engagement mediates differentiation of cardiac fibroblast precursor cells. Proc Natl Acad Sci USA 2008; 105: 10179-10184.

23 Castanio AP, Lin SL, Surowy T, Nowlin BT, Turlapati SA, Patel T, Singh A, Li S, Lupher $\mathrm{ML}$, Duffield JS. Serum amyloid P inhibits fibrosis through FCgR-dependen monocytemacrophage regulation in vivo. Sci Trans/ Med 2009; 1: 1-11.

24 Marvar PJ, Thabet SR, Guzik TJ, Lob HE, McCann LA, Weyand C, Gordon FJ, Harrison DG. Central and peripheral mechanisms of T-lymphocyte activation and vascular inflammation produced by angiotensin II-induced hypertension. Circulation Res 2010; 107: 263-270.

25 Qi G, Jia L, Li Y, Bian Y, Cheng J, Li H, Xiao C, Du J. Angiotensin II infusion-induced inflammation, monocytic fibroblast precursor infiltration, and cardiac fibrosis are pressure dependent. Cardiovasc Toxicol 2011; 11: 157-167.

26 Haudek SB, Cheng J, Du J, Wang Y, Hermosillo-Rodriguez J, Trial J, Taffet GE, Entman ML. Monocytic fibroblast precursors mediate fibrosis in angiotensin-II-induced cardiac hypertrophy. J Mol Cell Cardiol 2010; 49: 499-507.

$27 \mathrm{He} \mathrm{Z,} \mathrm{Way} \mathrm{KJ,} \mathrm{Arikawa} \mathrm{E,} \mathrm{Chou} \mathrm{E,} \mathrm{Opland} \mathrm{DM,} \mathrm{Clermont} \mathrm{A,} \mathrm{Isshiki} \mathrm{K,} \mathrm{Ma} \mathrm{RC,} \mathrm{Scott} \mathrm{JA,}$ Schoen FJ, Feener EP, King GL. Differential regulation of angiotensin II-induced expression of connective tissue growth factor by protein kinase $\mathrm{C}$ isoforms in the myocardium. J Biol Chem 2005; 280: 15719-15726.

28 Chen MM, Lam A, Abraham JA, Schreiner GF, Joly AH. CTGF expression is induced by TGF- beta in cardiac fibroblasts and cardiac myocytes: a potential role in heart fibrosis. J Mol Cell Cardiol 2000; 32: 1805-1819.

29 Wang X, McLennan SV, Allen TJ, Twigg SM. Regulation of pro-inflammatory and pro-fibrotic factors by CCN2/CTGF in H9c2 cardiomyocytes. J Cell Commun Signal 2010; 4: 15-23.

30 Ruperez M, Lorenzo O, Blanco-Colio LM, Esteban V, Egido J, Ruiz-Ortega M. Connective tissue growth factor is a mediator of angiotensin II-induced fibrosis. Circulation 2003; 108: 1499-1505.

31 Frenette PS, Wagner DD. Adhesion molecules-Part II: blood vessels and blood cells. N Engl J Med 1996; 335: 43-45.

32 Frenette PS, Wagner DD. Adhesion molecules-Part 1. N Engl J Med 1996; 334: 1526-1529.

33 Sallusto F, Mackay CR. Chemoattractants and their receptors in homeostasis and inflammation. Curr Opin Immunol 2004; 16: 724-731.

34 Olson TS, Ley K. Chemokines and chemokine receptors in leukocyte trafficking. Am J Physiol Regul Integr Comp Physiol 2002; 283: R7-28.

35 Luster AD. Chemokines-chemotactic cytokines that mediate inflammation. $N$ Engl J Med 1998; 338: 436-445.

36 Keeley EC, Mehrad B, Strieter RM. The role of circulating mesenchymal progenitor cells (fibrocytes) in the pathogenesis of fibrotic disorders. Thromb Haemost 2009; 101: 613-618.

37 Sakai N, Wada T, Yokoyama H, Lipp M, Ueha S, Matsushima K, Kaneko S. Secondary lymphoid tissue chemokine (SLC/CCL21)/CCR7 signaling regulates fibrocytes in renal fibrosis. Proc Natl Acad Sci USA 2006; 103: 14098-14103.

38 Bellini A, Mattoli S. The role of the fibrocyte, a bone marrow-derived mesenchymal progenitor, in reactive and reparative fibroses. Lab Invest 2007; 87: 858-870.

39 Chen J, Kuhlencordt PJ, Astern J, Gyurko R, Huang PL. Hypertension does not account for the accelerated atherosclerosis and development of aneurysms in male apolipoprotein e/endothelial nitric oxide synthase double knockout mice. Circulation 2001; 104: 2391-2394.

40 Paradis P, Dali-Youcef N, Paradis FW, Thibault G, Nemer M. Overexpression of angiotensin II type I receptor in cardiomyocytes induces cardiac hypertrophy and remodeling. Proc Natl Acad Sci USA 2000; 97: 931-936.

41 Watanabe D, Tanabe A, Naruse M, Morikawa S, Ezaki T, Takano K. Renoprotective effects of an angiotensin II receptor blocker in experimental model rats with hypertension and metabolic disorders. Hypertens Res 2009; 32: 807-815.

42 Shibata S, Nagase M, Yoshida S, Kawachi H, Fujita T. Podocyte as the target for aldosterone: roles of oxidative stress and Sgk1. Hypertension 2007; 49: 355-364. 
43 Cheng XW, Okumura K, Kuzuya M, Jin Z, Nagata K, Obata K, Inoue A, Hirashiki A, Takeshita K, Unno K, Harada K, Shi GP, Yokota M, Murohara T. Mechanism of diastolic stiffening of the failing myocardium and its prevention by angiotensin receptor and calcium channel blockers. J Cardiovasc Pharmacol 2009; 54: 47-56.

44 Nagata K, Obata K, Xu J, Ichihara S, Noda A, Kimata H, Kato T, Izawa H, Murohara T, Yokota M. Mineralocorticoid receptor antagonism attenuates cardiac hypertrophy and failure in low-aldosterone hypertensive rats. Hypertension 2006; 47: 656-664.

45 Habibi J, Demarco VG, Ma L, Pulakat L, Rainey WE, Whaley-Connell AT, Sowers JR. Mineralocorticoid receptor blockade improves diastolic function independent of blood pressure reduction in transgenic model of Raas overexpression. Am J Physiol 2011; 300: H1484-H1491.

46 Rey S, Semenza GL. Hypoxia-inducible factor-1-dependent mechanisms of vascularization and vascular remodelling. Cardiovasc Res 2010; 86: 236-242.
47 Knowles HJ, Tian YM, Mole DR, Harris AL. Novel mechanism of action for hydralazine: induction of hypoxia-inducible factor-1alpha, vascular endothelial growth factor, and angiogenesis by inhibition of prolyl hydroxylases. Circulation Res 2004; 95: 162-169.

48 Lin M, Chiang HT, Lin SL, Chang MS, Chiang BN, Kuo HW, Cheitlin MD. Vasodilator therapy in chronic asymptomatic aortic regurgitation: enalapril versus hydralazine therapy. J Am Coll Cardiol 1994; 24: 1046-1053.

49 Schmieder RE, Schlaich MP, Klingbeil AU, Martus P. Update on reversal of left ventricular hypertrophy in essential hypertension (a meta-analysis of all randomized double-blind studies until December 1996). Nephrol Dial Transplant 1998; 13: 564-569.

50 Greenberg B, Massie B, Bristow JD, Cheitlin M, Siemienczuk D, Topic N, Wilson RA, Szlachcic J, Thomas D. Long-term vasodilator therapy of chronic aortic insufficiency. A randomized double-blinded, placebo-controlled clinical trial. Circulation 1988; 78 : 92-103. 